\title{
IMPLICATIONS OF MACROPHAGE T-LYMPHOCYTE INTERACTIONS FOR TUMOR REJECTABILITY
}

\author{
Rob J. de Boer and Pauline Hogeweg \\ BIOINFORMATICS GROUP \\ University of Utrecht \\ Padualaan $8,3584 \mathrm{CH}$ Utrecht \\ The Netherlands
}

\begin{abstract}
A relatively detailed model of experimentally described macrophage T-lymphocyte interactions has been developed. In this model we investigate the immune response to tumors that differ in antigenicity and/or in initial size. Having deliberately omitted from the model tumor escape mechanisms (e.g. suppression, antigenic modulation or heterogeneity), we study the circumstances that nevertheless lead to progressive tumor growth.

The model behavior shows that: (1) tumor antigenicity can best be defined in terms of helper $T$ cell reactivity; (2) small differences in the availability of HTL (*) markedly influence tumor rejectability; (3) compared with the impact of macrophages, the impact of cTL increases more with increasing tumor antigenicity; and (4) sneaking through and tolerance are intrinsic to this model.

HTL have a large impact on the model behavior (i.e. the immune response) because there are self-reinforcements in the HTL activation and proliferation process. Interestingly, unresponsiveness (tolerance) evolves in this model, despite the presence of these se1f-reinforcements and the absence of negative interactions (e.g. suppression). Tolerance is caused by a proliferation threshold that comes into existence when T-1ymphocyte effectors are made short-1ived. We discuss the advantages of using numerical integration combined with numerical phase state analysis. Stable steady states in this model do exist but are of minor importance.
\end{abstract}

\footnotetext{
ABBREVIATIONS: ANGRY cytotoxic macrophage(s), APC antigen presenting ce11(s), CTL cytotoxic T-1ymphocyte(s), CTLP CTL-precursor(s), HTL he1per T-1ymphocyte(s), HTLP HTL-precursor(s), IFN interferon, IL 2 interleukin 2, MAF macrophage activating factor, MPH normal macrophage(s), NK natural killer cell(s).
} 


\section{INTRODUCTION}

Immune reactions usually involve interactions between a large number of cel1 types. Mathematical models in immunology are however normally simplifications, representing only part of the available immunological data. Two types of model simplifications should be distinguished: those imposed by the modelling formalism (and the method of analysing the model) and those imposed for reasons of informatic minimalization. In order to obtain insight into the dynamics of complex systems, the former type of simplification should be minimized, whereas the latter type should be maximized.

Models that incorporate only part of the data, i.e. cell-interactions experimentally known to exist, exhibit behavior that corresponds to experimentally known phenomena. The investigation of the relationships between the set of incorporated interactions (i.e. the micro level) and the model behavior (i.e. the macro 1eve1) [1] provides insight into the role of the various processes that are involved in immune reactions. Moreover, it is possible to pinpoint the "key interactions" responsible for generating a specific phenomenon if al1 redundant interactions are removed, i.e. a model is simplified $(\mathrm{e} \cdot \mathrm{g} \cdot[2])$.

Here we investigate immune responses to tumors, i.e. antigens capable of endless replication. Many different effector cells are known to play a role in the immune resistance to tumors: e.g. NK cells, $B$ cells, helper $T$ cells, cytotoxic $T$ cells and macrophages [3]. 0ur model specifies only a subset of these cells (i.e. helper and cytotoxic $T$ cel1s and macrophages), and only one compartment is taken into consideration. The interactions between the incorporated cell types are however specified in a relatively "knowledge oriented" way. Interestingly, the behavior of the model is diverse, and corresponds to a number of phenomena described experimenta11y.

We have incorporated the following data in the model (see Table 1 and Fig. 1): a) small tumors grow exponential1y [4], large ones linearly; b) T-1ymphocytes and macrophages can become cytotoxic towards tumor cells [3]; c) macrophages process tumor cell debris (which accumulates upon normal tumor cell death and upon tumor cell lysis), and present the processed tumor associated antigens in an antigenic form [5]; d) HTLP activation requires antigen presentation, whereas CTLP activation does not [6]; e) CTL and HTL are capable of proliferation (in response to IL2) [7, 8], whereas macrophages are not [9]; f) CTLP maturation into the CTL stage requires the presence 


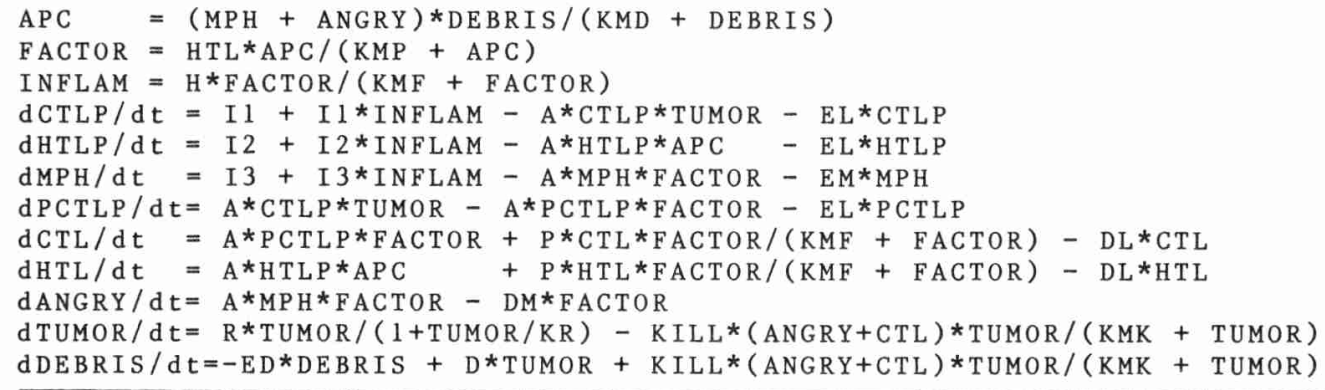

Table 1. The cell interactions specified by the model. Tumor cell killing is effected by two effector cells: cytotoxic T-1ymphocytes (CTL) and cytotoxic macrophages (ANGRY). CTL precursors (CTLP) require both activation by antigen (TUMOR) and stimulation by lymphoid factor (FACTOR) before they transform into cytotoxic effector cells, which are capable of proliferation. Transformation of macrophages (MPH) into their cytotoxic effectors (ANGRY) is caused by lymphoid factors (FACTOR) released by activated helper T cells (HTL) upon restimulation of the latter by antigen presenting cells (APC). Only HTL produce lymphoid factors; the different factors are assumed to be kinetically identical and are combined into one variable (FACTOR). HTL precursors (HTLP) become activated (effectors) upon interaction with APC. Effector T-lymphocytes (CTL, HTL) proliferate in response to interleukin 2 (FACTOR); effector macrophages on the other hand can only be formed from their precursors. The influx of precursors is increased with INFLAM during an inflammation reaction. The intensity of the inflammation reaction depends on the concentration of lymphoid factors (FACTOR). Effector restimulation (KMP), proliferation stimulation (KMF) and tumor cell killing (KMK) [49] follow conventional Michaelis-Menten kinetics.

Figure 1. The interactions incorporated in the model.

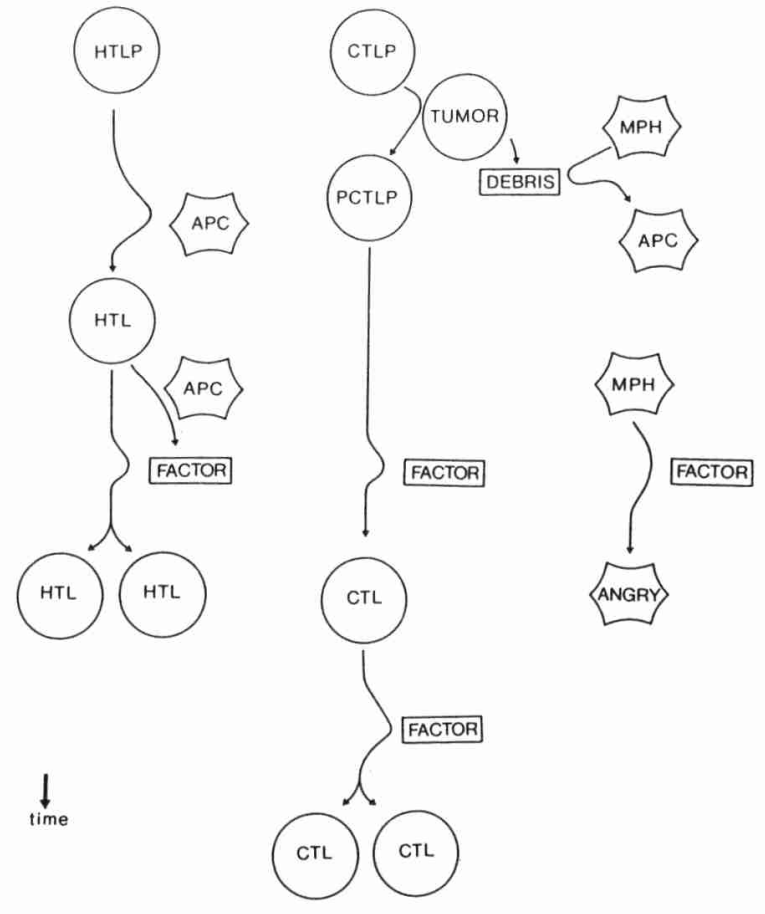




\begin{tabular}{|c|c|c|c|c|c|c|c|}
\hline A & $10^{-3}$ & activation rate & per $c \in$ & $11 \mathrm{p}$ & er da & & \\
\hline $\mathrm{D}$ & 0.1 & DEBRIS generation rate & units & per & ce11 & per & day \\
\hline DL & 0.02 or 0.2 & 1ymphocyte effector decay & per $d a$ & & & & \\
\hline DM & 1.0 & cytotoxic macrophage decay & per da & & & & \\
\hline ED & 2.0 & debris decay & per da & & & & \\
\hline EL & 0.02 & 1ymphocyte precursor efflux & per & & & & \\
\hline EM & 0.05 & normal macrophage efflux & per da & & & & \\
\hline $\mathrm{H}$ & 9.0 & inf 1 ammation constant & & & & & \\
\hline I 1 & 1.0 or 200 & CTLP influx & cel1s & per & day & & \\
\hline I 2 & 0.01 to 1000 & HTLP influx & $\operatorname{ce} 11 \mathrm{~s}$ & per & day & & \\
\hline I 3 & 125000 & macrophage influx & $\operatorname{ce} 11 \mathrm{~s}$ & per & day & & \\
\hline KILL & 10,0 & ki11ing capacity & $\operatorname{cel} 1 \mathrm{~s}$ & per & ce 11 & per & day \\
\hline KMD & $10^{7}$ & presentation saturation & units & & & & \\
\hline KMF & 50.0 & factor saturation & units & & & & \\
\hline KMK & 105 & killing saturation & cel1s & & & & \\
\hline KMP & $10^{3}$ & restimulation saturation & $\operatorname{ce} 11 \mathrm{~s}$ & & & & \\
\hline $\mathrm{KR}$ & $10^{9}$ & growth rate saturation & ce11s & & & & \\
\hline $\mathrm{P}$ & 1.0 & proliferation rate & ce $11 \mathrm{~s}$ & per & ce 11 & per & day \\
\hline R & 1.0 & tumor growth rate & ce $11 \mathrm{~s}$ & per & ce 11 & per & day \\
\hline
\end{tabular}

Table 2. The parameter setting of the model is based upon experimental data concerning the immune resistance of DBA/2 mice to the SL2 tumor after the tumor has been injected into the peritoneal cavity of the mice [20]. The parameter values were discussed previously in more detail [14]. The degree of tumor antigenicity is defined as the number of 1 ymphocyte precursors that can be activated upon introduction of the tumor, i.e. antigenicity corresponds to T-1ymphocyte influx (II and I2). In order to represent different tumors I1 and I2 are varied. We study the effect of T-lymphocyte effector longevity (DL) by changing it 10-fold.

of a lymphoid differentiation factor [10, 11]; and g) upon antigenic restimulation HTL produce lymphoid factors: T cel1 growth factor (IL2) [7, 8], T cel1 differentiation factor [10, 11], macrophage activating factor (MAF, IFN) [12], and factors inducing an inflammation reaction [13]. This model is an extension of models described in more detail previously [14, 15]. Here HTL are restimulated by APC (instead of by TUMOR) and CTL induction requires the presence of HTL-derived differentiation factors.

For the informatic minimalization reasons mentioned above, only stimulating (positive) interactions have been incorporated in our mode1. Suppressor cells (of $\mathrm{T}$ cell or of macrophage origin [16, 17]) however "down regulate" anti-tumor immune responses (i.e. influence them negatively). In order to investigate whether the failure of immune responses hinges upon suppression and/or other tumor escape mechanisms (e.g. antigenic heterogeneity [18] or modulation [19, Michelson: this volume]) we have omitted these mechanisms. 
The experimental system upon which this model and its parameter setting (Table 2) are based on the ascitic growth of the sL2 tumor in the peritoneal cavity of DBA/2 mice [20, 21]. In this paper we describe (theoretical) experiments with tumors that differ in antigenicity. To this end we define the degree of antigenicity as the initial sizes of the T-lymphocyte precursor populations that can be activated upon introduction of that antigen.

It turns out that (minimal differences in) tumor antigenicity can determine whether rejection or progressive tumor growth occurs (Fig. 4), i.e. can determine the failure of the response. Progressive tumor growth can either be accompanied by an ever increasing T-1ymphocyte response (Fig. 3 and $8 \mathrm{c}$ ) or by a constant and very limited reaction (Fig. 8a). Suppression, which is absent, is thus redundant for the failure of the response.

METHODS

We investigate our model by introducing tumors of varying antigenicity and/or tumors in various initial doses. These model immune systems provide a more advantageous environment for experimenting than do wet immune systems because a) all variables are easily observable, and b) the system structure can be manipulated easily (i.e. the impact of different processes can be studied through their incorporation or omittance).

The model is formulated in ordinary differential equations. APC, FACTOR and the inflammation reaction are incorporated in the form of quasi steady state variables. The model is studied by means of numerical integration (i.e. by simulation). Analytical methods would put more severe constraints on the complexity of the model immune system. Furthermore analytical methods usually concentrate on the existence of steady states, which are not necessarily of prime interest.

The power of the simulation method is augmented by static analysis of the state space. We obtain insight into the qualitative differences in the model behaviour by static (graphical) analysis. We reduce the 9-D state space of the model (there are 9 variables) to various $3-D$ or $2-D$ state spaces by making quasi steady state assumptions for the other ( 6 or 7 ) variables. It turns out that such state spaces with 0-isoclines provide a valuable tool for the 
interpretation of the model behavior. For instance, specific phenomena can be related to specific forms (folds) of the 0-isocline planes.

The model is investigated by means of GRIND [22]. GRIND performs numerical searches for 0-isoclines; it integrates by means of RoW4A [23], which is a robust integrator for the analysis of stiff systems of ordinary differential equations.

RESULTS AND CONCLUSIONS

antigenicity. In Fig. 2 the model immune system is challenged with a tumor consisting of one cell and having an antigenicity corresponding to $I 1=1.0 \quad(\operatorname{CTLP}=50)$ and I $2=0.2$ (HTLP=10). In Fig. 3 the same system is challenged with a tumor of equal size but which is slightly less antigenic (i.e. $I 1=1.0$ and $I 2=0.1 \quad($ HTLP=5)). The former tumor (Fig. 2) is rejected, whereas the latter (Fig. 3) grows in an uncontrolled manner. We therefore conclude that minimal differences in HTLP reactivity (here 5 cells, see Fig. 4) can markedly influence tumor rejectability.

The immune reactions to these two tumors are very similar up till day 10. At day 10 , the CTLP have been transformed into PCTLP by activation, the PCTLP remain constant (the availability of differentiation factor limits their maturation into the CTL stage), some DEBRIS has accumulated, HTL induction has just started, and a few hundred ANGRY macrophages have been induced. In comparison to the "progressive growth" case (Fig. 2), the TUMOR is $1 \%$ smaller in the rejection case (Fig. 3), DEBRIS is 6\% larger, ANGRY and HTL are about twice as large, and PCTLP is roughly equal. Note that the initial (at day zero) HTLP populations also differ by a factor two. HTL proliferation is a self-reinforcing process because these cells produce their own growth factor (IL2). The difference in the HTL numbers of the two immune reactions therefore increases (up till day 15, when the tumor is rejected). Moreover, HTL activation is also self-reinforcing: HTLP activation requires the accumulation of DEBRIS, the HTL thus generated induce ANGRY macrophages, which in turn increase the accumulation of DEBRIS by lysing tumor cells. In addition, the production of IL2 and MAF (FACTOR) increases upon an increase in DEBRIS, since HTL depend on antigen presentation for restimulation. These self-reinforcements in the HTL dynamics explain why minor changes in HTLP reactivity have a major impact on tumor rejectability. 


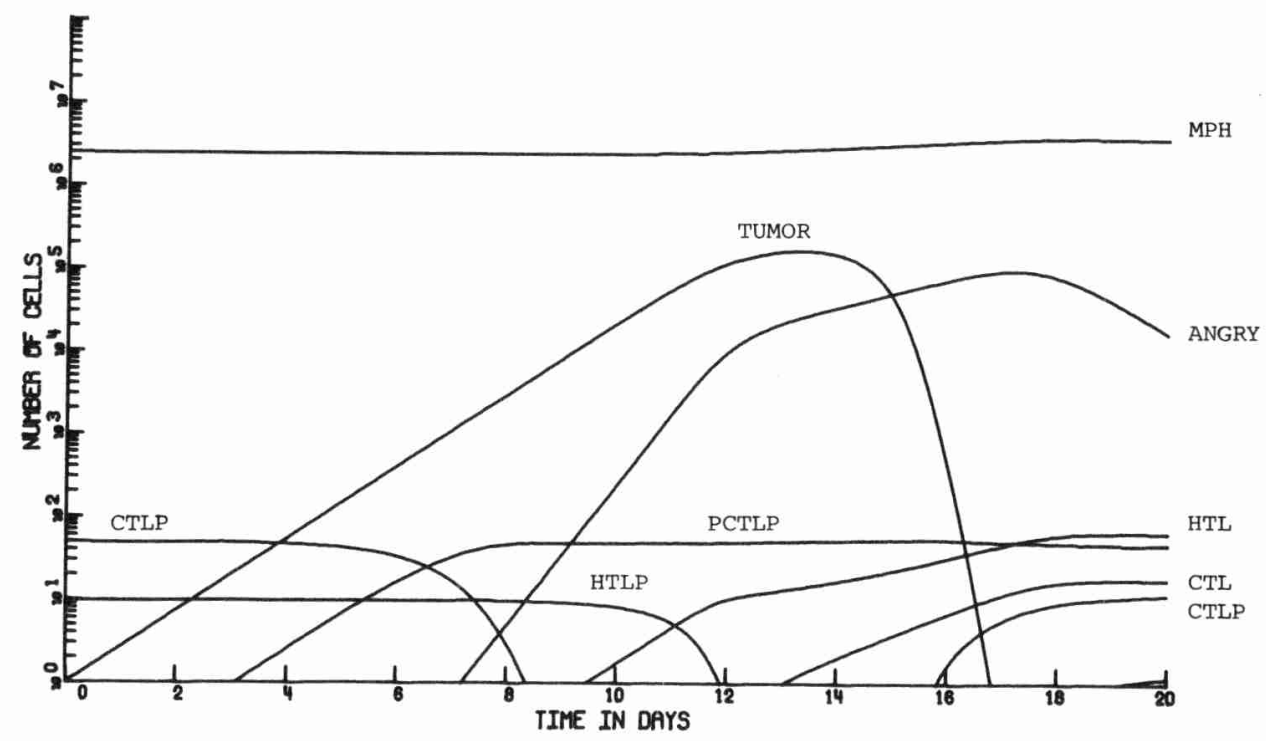

Figure 2. The rejection of a tumor of an antigenicity correponding to I $1=1.0$ (CTLP=50) and I 2=0.2 (HTLP=10) introduced as a single cell into a non-immunized system (day 0 ). Parameters as in Table 2, DL $=0.02$.

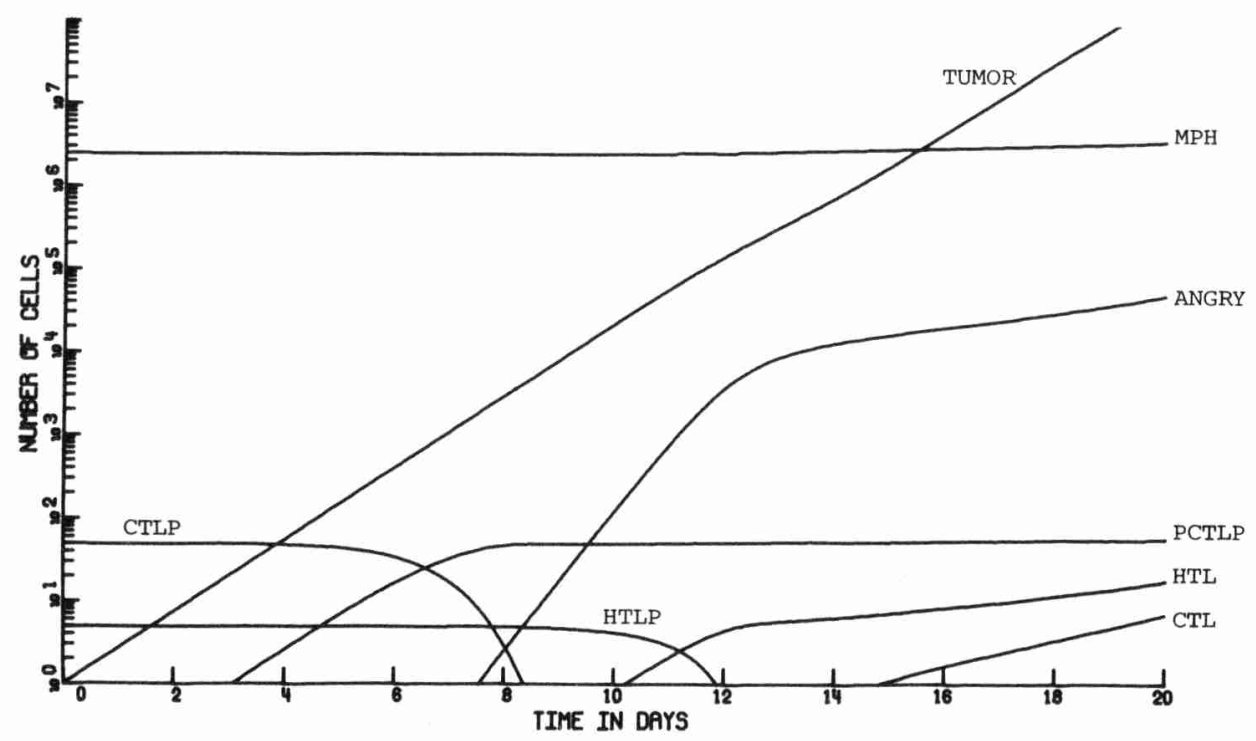

Figure 3. The progressive growth of a tumor corresponding to Il=1.0 ( CTLP=50) and I $2=0.1 \quad($ HTLP=5) introduced as a single cell at day 0 . Parameters as in Table $2, \mathrm{DL}=0.02$. 
The general relation between antigenicity and rejectability is depicted in Fig. 4. The figure shows that weakly antigenic tumors cannot be rejected whatever their initial size, whereas tumors that are slightly more antigenic can be rejected even if they are introduced in a large dose. Hundred-fold variations in CTLP reactivity do not change the form and position of the graph in Fig. 4. As was concluded before for our previous models [14], tumor antigenicity can thus best be defined as helper $T$ cell reactivity. Experimentally tumor antigenicity is usually defined as the size of the largest rejectable tumor, which is in our analysis a very insensitive parameter.

Weakly antigenic tumors that escape (e.g. Fig. 3) do not however evoke weaker immune reactions. At day 24 of the simulation shown in Fig. 3 all effector populations are larger than the maximum populations reached in the rejection case (Fig. 2). However, because the tumor is large $\left(>10^{9}\right.$ cells) at that time these effectors have little effect. These results are in close correpondance with the data of Lannin et al. [24], who show that the T-lymphocyte response to a fibrosarcoma is "too little and too slow" for tumor rejection.

Helper $T$ cells play a crucial role in the immune reaction of the mode1. Helper reactivity for instance determines tumor rejectability (Fig. 4). Moreover, otherwise lethal tumors can be rejected when the

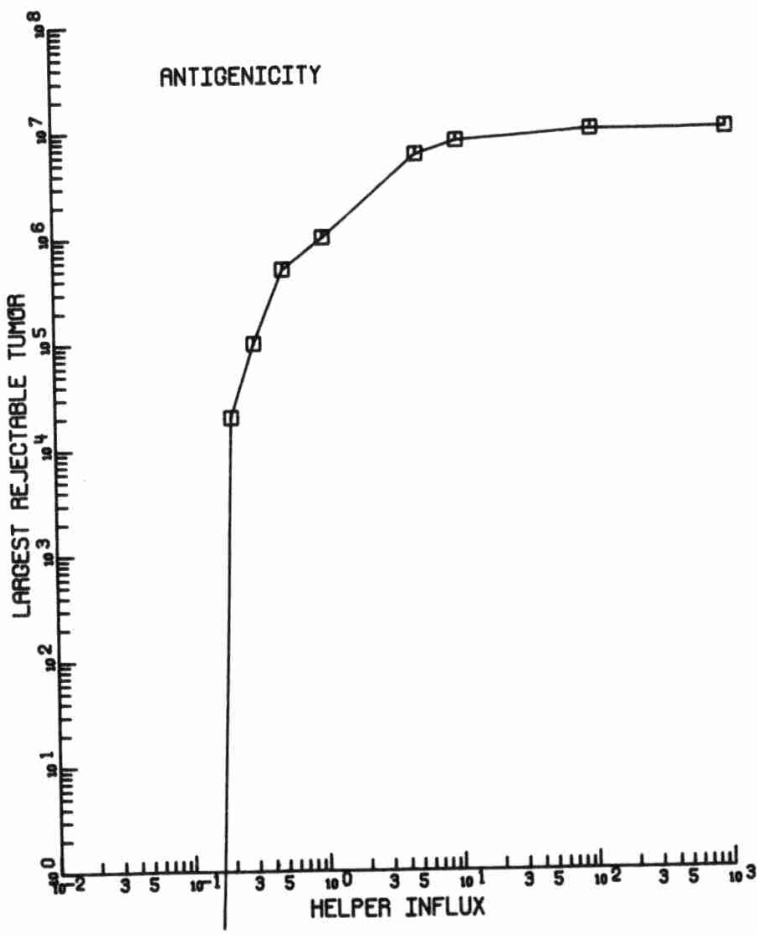

Figure 4. The relation between tumor rejectability (i.e. the size of the largest rejectable tumor) and tumor antigenicity (i.e. HTLP reactivity). The tumors range in antigenicity from I $2=0.01$ (HTLP=0.5) to $\quad$ I $2=1000$ (HTLP $=50,000)$. Parameters as in $\mathrm{Table} 2, \mathrm{DL}=0.02$. 
model is previously immunized with that tumor, due to an increase in HTL numbers [14]. In addition, adoptive transfer of 5 HTL at day zero enables the current model to reject the lethal tumor of Fig. 3 . The effect that HTL have on the model behavior is depicted in Fig. 5. At low HTL numbers ( $i . e$. at the back of the cube) the ANGRY isocline lies entirely below the TUMOR'=0 isocline, whereas at high HTL numbers (at the front) the largest part of the ANGRY isocline lies above the TUMOR
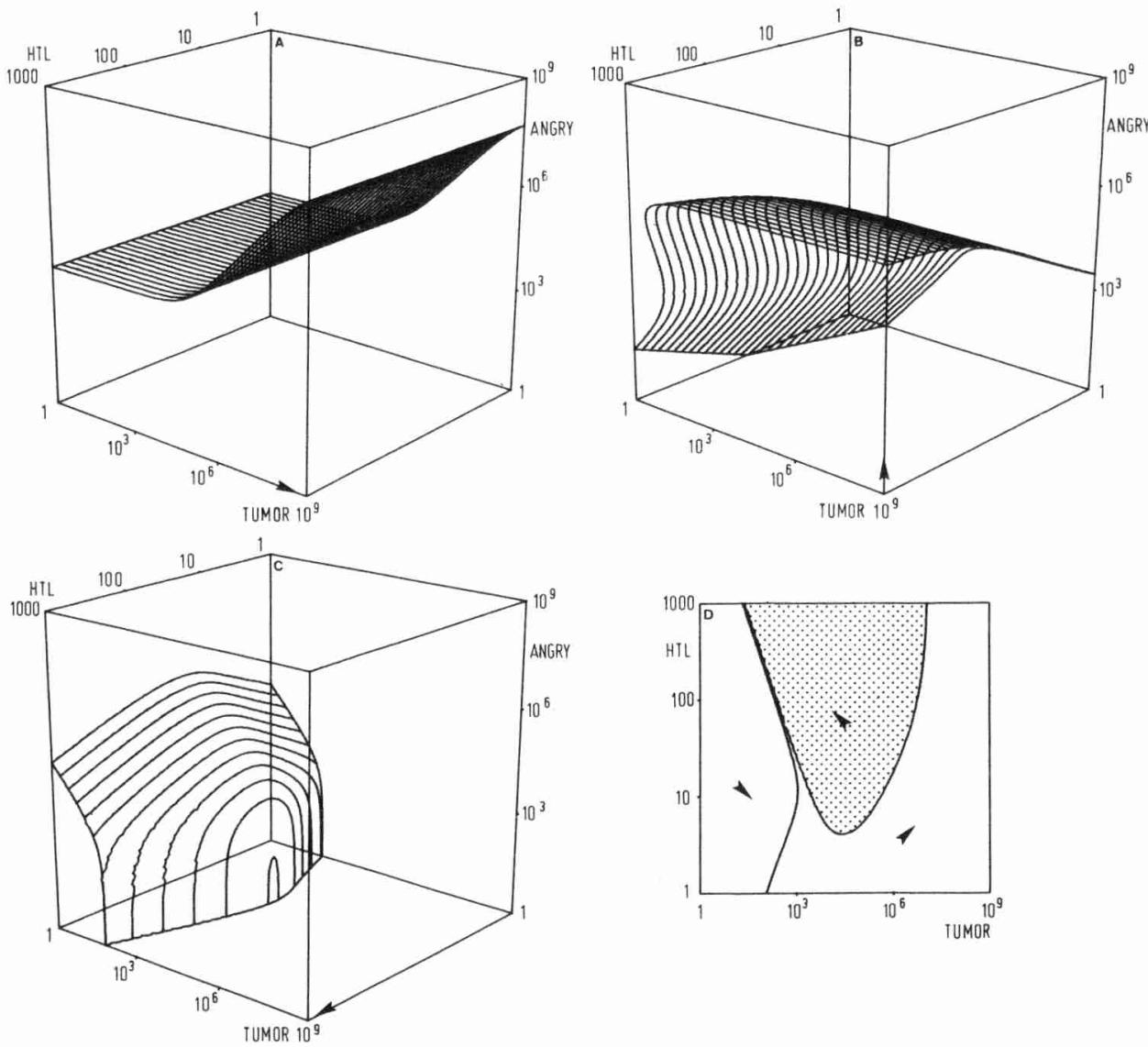

Figure 5. Phase portrait of the model challenged with the tumor of Fig. 3 (rejection). Assuming CTLP, HTLP, MPH, PCTLP, CTL and DEBRIS at their respective (positive) quasi steady state values, we depict the TUMOR $=0$ (A), the ANGRY $=0$ (B) and the HTL ${ }^{\prime}=0$ (C) isocline planes. The arrows indicate the local direction of trajectories. In Fg. 5d ANGRY is also in quasi steady state, the region corresponding to TUMOR'<0 (tumor regression) is shaded in that figure. Parameters as in Table 2 , I $1=1.0$, I $2=0.2, \mathrm{DL}=0.02$. 
isocline. Therefore, at the front there is a large region in which TUMOR decreases and ANGRY increases (which corresponds to tumor regression). At the back however trajectories never ascend into the TUMOR'<0 region because ANGRY numbers cannot become 1 arge enough there. Tumor rejection is hence impossible at low HTL numbers. In the 2-D phase portrait (Fig. 5d), where ANGRY is at quasi steady state, it is evident that tumor regression (i.e. the shaded region) is only possible at high HTL numbers. The figure ( 5 b, d) also shows that the macrophage response cannot grow infinitely large, so large tumors always increase (large tumor trajectories move to the right along the horizontal part of the ANGRY isocline (Fig. 5b)).

effector switch. In contrast to the "precursor bound" macrophage response [2] the T-1ymphocyte response is "proliferative" [15] because it can become infinitely large by repeated T-1ymphocyte proliferation. Tumors that are too large to be rejected by macrophages can therefore be rejected by the CTL population if the latter proliferates (provided the tumor population does not have a faster per capita growth rate than the CTL population). However, for the tumor depicted in Fig. 3, CTL proliferation into a large effector population takes such a long time that the tumor meanwhile has killed the mouse. (since mice die from tumors of about $10^{8}$ cells [25], we stop the simulations around that size). Highly antigenic tumors on the other hand correspond, by definition, to large T-1ymphocyte precursor populations, which require fewer cell divisions (i.e. less time) to reach the size required for tumor rejection. In Fig. 6 we show such a case. Note that smaller doses of this highly antigenic tumor (i.e. I $1=200$, I $2=10$ ) would easily be rejected by the ANGRY macrophages.

About $10^{8} \mathrm{CTL}$ and $10^{7} \mathrm{HTL}$ are present at the time of tumor rejection (day 10). For both populations this roughly corresponds to a 10,000-fold increase (T-1ymphocyte populations are reported to be able to expand to this extent [26]). After the rejection of the tumor, proliferation however continues because a large amount of DEBRIS has accumulated; DEBRIS is removed relatively slowly. The removal of DEBRIS upon phagocytosis by macrophages has been omitted from this model for simplicity. Moreover, we have ignored absorption of IL 2 by the proliferating ce11s; if IL2 absorbtion were incorporated the IL2 concentration would decrease faster after tumor rejection. Prolonged proliferation after tumor rejection is probably unrealistic.

In general however, this result (i.e. the predominance of CTL in reactions to large highly antigenic tumors) does correspond to the experimental data. Ishil et al. [27] show that the T-1ymphocyte infiltrate of methylcholanthrene-induced sarcomas increases with 


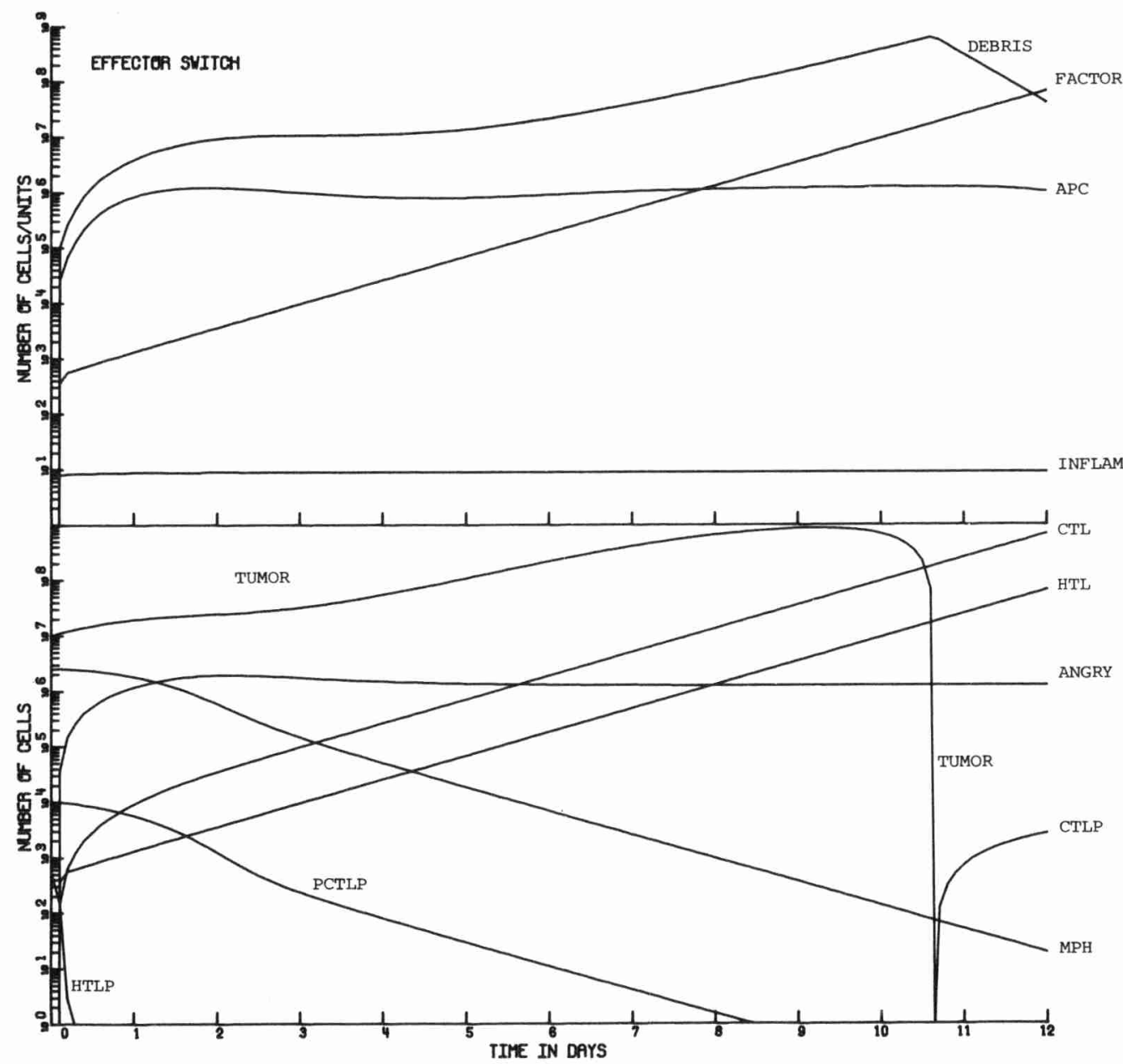

Figure 6. The rejection of an highly antigenic tumor, i.e. Il=200 $\left(\operatorname{CTLP}=10^{4}\right)$ and I $2=10\left(\right.$ HTLP=500). A large graft of this tumor $\left(10^{7}\right.$ cel1s) is introduced at day 0 into a non-immunized system (a smaller dose would be rejected by macrophages). Parameters as in Table 2, $\mathrm{DL}=0.02$.

increasing antigenicity of the tumor, whereas the macrophage infiltrate remains grossly equal. CTL are reported to play a significant role in the immune resistance to virus-induced, i.e. highly antigenic, tumors [3]. The current model also accounts for the experimental fact that immunization mainly increases the T-lymphocyte part of the anti-tumor immune response [28]. Immunization increases the number of T-1ymphocytes, which consequently require fewer cell divisions (i.e. less time) to become abundant.

Fig. 7 shows the TUMOR $=0$ isocline plane in a TUMOR, HTL, and 
Figure 7. Phase portrait of the mode1. In a TUMOR, CTL, HTL state space we depict the TUMOR $=0$ isocline plane. CTLP, HTLP, MPH, PCTLP, ANGRY and DEBRIS are assumed to be in (positive) quasi steady state. The arrows indicate the local direction of trajectories. The form and the position of this plane are independent of tumor antigenicity (i.e. I 1 and I2) because both T lymphocyte effectors (HTL and (TL) are part of the state space. The figure thus represents all tumors studied hitherto. Parameters as in Table 2, $\mathrm{DL}=0.02$.

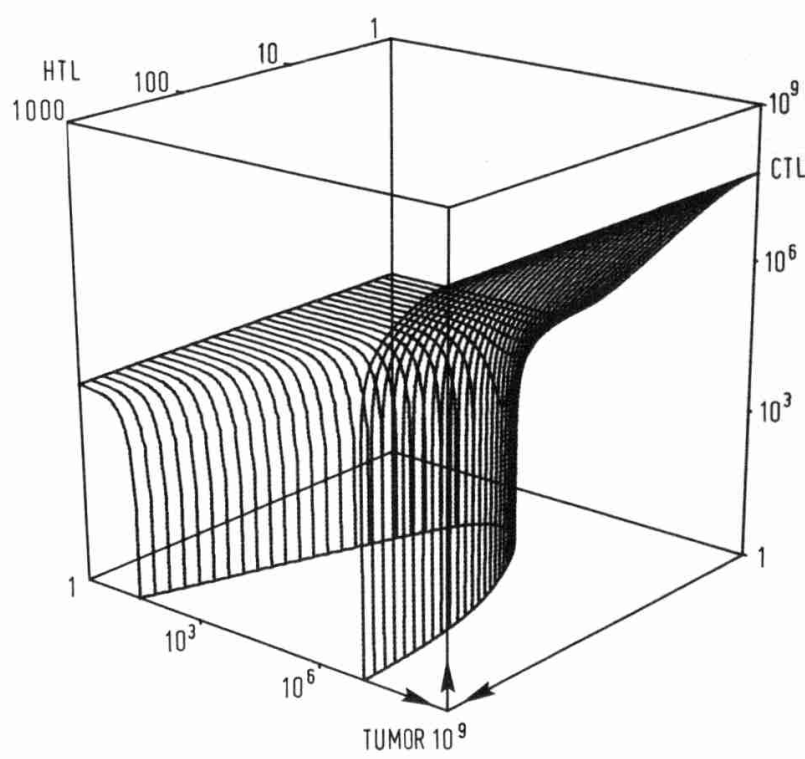

CTL state space (ANGRY and all other variables are in quasi steady state). At high HTL numbers (and hence high ANGRY numbers) intermediate sized tumors regress (i.e. in the central valley), 1arger tumors however only decrease at high CTL numbers (i.e. above the p 1 ane).

sneaking through and low zone tolerance. Although most of the parameters of the model were taken from the 1iterature, see Ref. [14], several had to be filled in arbitrarily. The lifespans of the activated T-1ymphocytes (HTL, CT1, PCTLP) are amongst the parameters that were chosen arbitrarily. Experimentally these cells are known to be short-lived as effector cells but they are also known to be long-lived as "memory" cells. The processes that determine T-1ymphocyte 1ongevity are largely unknown [29]. In the previous simulations (Fig. 2-7) the rate of T-1ymphocyte effector decay was (simply) chosen identical to that of their precursors (i.e. 50 days). In this section however we choose to make the T-1ymphocyte effectors (HTL, CTL) short-lived ( 5 days); all other parameters are left the s ame.

The longevity of T-1ymphocyte effectors has a profound effect on the model behavior. An example is illustrated in Fig. 8. This tumor (having an antigenicity corresponding to $I 1=1.0$, I2=0.3) grows progressively (i.e. sneaks through) when it is introduced in a small dose (e.g. 1 cell, Fig. 8a), it is rejected when introduced in intermediate doses (e.g. $10^{4}$ cel1s, Fig. $8 \mathrm{~b}$ ), and it grows 


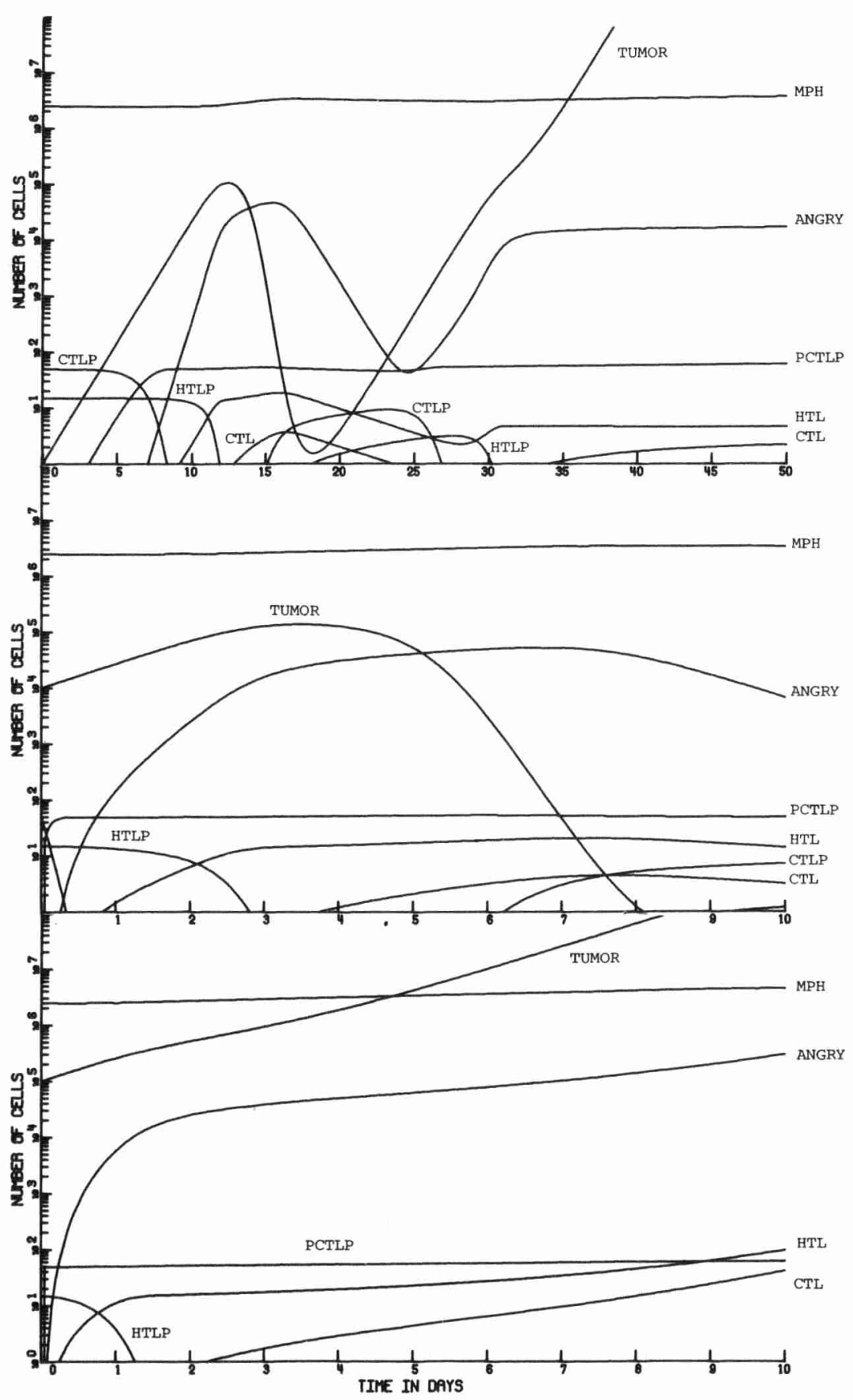

Figure 8. The sneaking through phenomenon. A tumor corresponding to $I l=1.0$ and $I 2=0.3$ is introduced as a single ce11 (A), in a dose of $10^{4}$ cells (B), and in a dose of $10^{5}$ cells (C). Only the intermediate sized tumor can be rejected (B). Parameters as in Table 2, DL=0.2. 


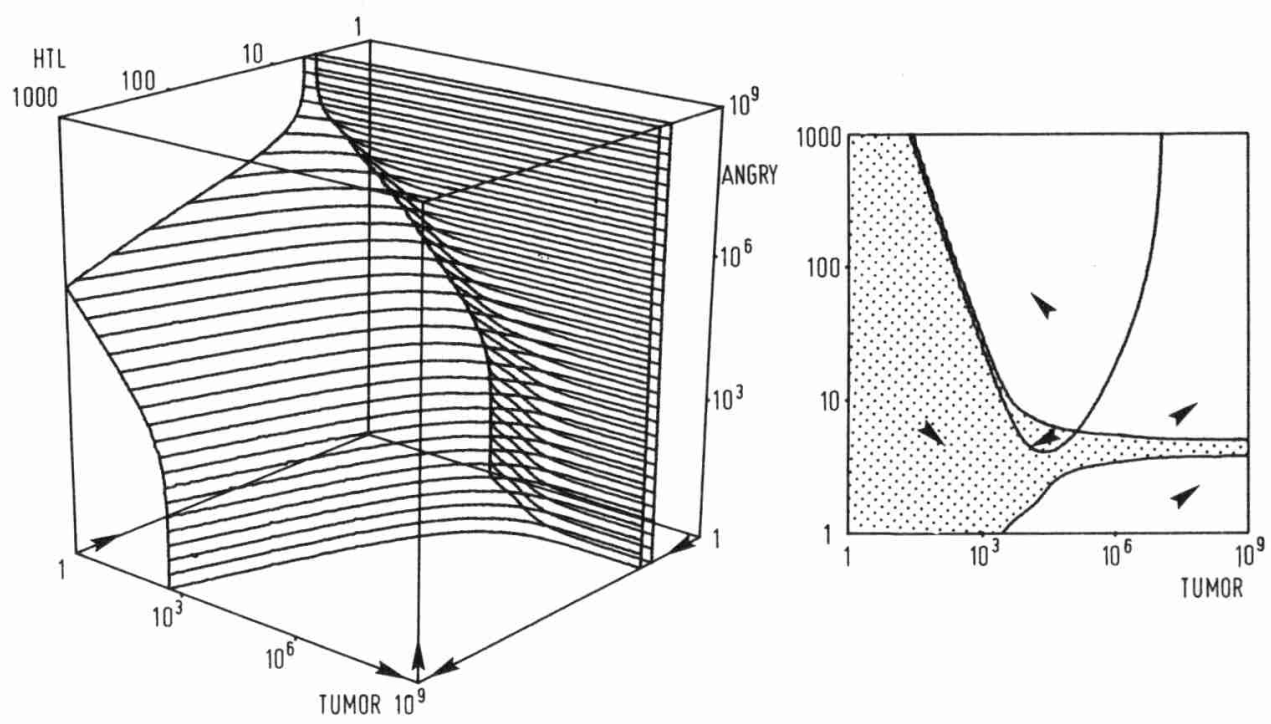

Figure 9. Phase portrait of the sneaking through phenomenon. All variables not depicted in the figure are assumed to be at quasi steady state. The HTL ${ }^{-}=0$ isocline separates the state space into distinct regions of HTL increase; the HTL'<0 region is shaded in Fig. 9 b. The arrows indicate the local direction of trajectories.

progressively again ( $i . e$. breaks through) when it is introduced in a large dose (e.g. $10^{5}$ cells, Fig. 8c). Such behavior is known experimentally as the sneaking through phenomenon: the progressive growth of initially small tumors in circumstances where larger tumors are rejected [30]. Immunization with the same tumor enhances the sneaking through behavior of the model (i.e. increases the range of tumor doses in which sneaking through occurs) [15], which is in agreement with the experimental data [31].

The progressive growth of the tumor introduced in a large dose (Fig. $8 \mathrm{c}$ ) is accompanied by an ever increasing fmmune reaction involving extensive T-1ymphocyte proliferation. The progressive growth phase of the initially small tumor (Fig. 8 a) by contrast, corresponds to a constant response of limited magnitude. Expansion of the antigen (the tumor) does not result in an increase of the immune response. This is known experimentally as tolerance or unresponsiveness. In this model sneaking through thus corresponds to low zone tolerance, $1 . e$. tolerance arising upon the introduction of very small doses of antigen [32, 33]. Prehn [30] argued that sneaking through could not be due to (low zone) tolerance because experimentally it was known to be enhanced 
by immunization [31]. The present form of sneaking through however is enhanced by immunization and does correspond to low zone tolerance.

The process whereby sneaking through (tolerance) evolves in this model can be revealed by examination of the phase plots in Fig. 9. The figure shows that HTL populations increase when they are small, decrease at intermediate HTL numbers (shaded in 9 b), and increase again at large HTL numbers. Thus although the HTL population is capable of infinite growth it first has to bridge a region in which it decreases. We define the minimum size of the HTL population required for continuous increase by proliferation as the proliferation threshold. The proliferation threshold corresponds to the HTL' $=0$ isocline that is situated at high HTL numbers. If effector cells are long-lived, the proliferation threshold, and hence sneaking through behavior, is absent (Fig. $5 \mathrm{c}, \mathrm{d})$.

HTL proliferation depends on the IL2 concentration ( $i . e$. depends on the size of the HTL populations), and is thus a self-reinforcing process. HTL decay however is independent of the presence of other HTL. It is therefore evident (if the maximum proliferation rate exceeds the decay rate) that large (stimulated) HTL populations increase. Small HTL populations, on the other hand, produce only a little IL2, and, as a consequence, proliferate slowly. If these cells are short-1ived, the slow proliferation rate will be outweighed by the decay rate. This corresponds to population decrease.

In this model tolerance is thus intrinsic to the kinetics of IL 2 production. Small doses of antigen induce low IL 2 concentrations because few effectors are activated. As a consequence these effectors decay without much proliferation. By the time the antigen has grown large enough to be able to activate a large number of precursors concomittantly (i.e. large enough to induce high IL2 concentrations), the precursor population has been reduced considerably due to the previous activations. If activation of the remaining precursor population is insufficient to generate an effector population larger than the proliferation threshold, the system is unresponsive (tolerant). Precursor depletion (here by activation) thus disables the system to such an extent that it is never again able to mount immune responses to that antigen. Precursor depletion was previously shown to be responsible for sneaking through in precursor bound models ( $i . e$. systems that specify proliferation as a "once only" occurence) [2]. The proliferation threshold makes the current proliferative T-1ymphocyte system precursor bound when it is slowly activated.

These results are in close correspondence with recent experimental data [34, 35], which show that immunological activation in 
the absence of IL2 results in tolerance.

In fact, it has been repeatedly shown that antigenic stimulation (signal 1 ) in the absence of helper $\mathrm{T}$ cel1 factors (signal 2) results in tolerance [36, 37, 38]. We show here that the absence of signal 2 (FACTOR) can be caused by slow activation, and, moreover, that a high turnover rate of effector cells suffices for the generation of tolerance.

\section{DISCUSSION}

methodology. In this paper we have concentrated on the dynamic behavior of the model, and not on the existence of steady states. We have shown that small differences in the mode1's initial state can markedly influence the dynamic behavior. For instance, the (artificial) introduction of $5 \mathrm{HTL}$ at day zero (i.e. adoptive transfer) changes the progressive tumor-growth behavior into tumor rejection behavior. In addition, the results on sneaking through show that the initial antigen dose can be of crucial importance for the outcome of the immune response (this is in fact one of the most interesting results).

Furthermore it is dynamically important that we stop the simulations whenever the tumor becomes smaller than one cell (i.e. tumor rejection) and whenever it grows too large (which corresponds to death of the host). For instance, the immune reactions to the tumors of Fig. 2 and 3 have a stable equilibrium at CTL=7050, ANGRY=2950, and HTL=4876, TUMOR=1.3 and HTL=2551, TUMOR=2.5 respectively. Both steady states are however only reached (a) after an unrealistically long time $(>100,000$ years), and (b) either via extremely small (Fig. 2, after "tumor rejection") or via extremely large (Fig. 3, after day 20) tumor sizes. These steady states are very similar (they differ by a factor two in HTL and TUMOR numbers upon a 2-fold change in antigenicity), but the corresponding dynamic behavior of the model is very different ( $1 . e$. rejection versus progressive tumor growth). It thus turns out that biologically meaningful results could only be obtained by studying the dynamic behavior; the steady states of these models are of minor (biologica1) importance.

Numerical integration (simulation) is the only tool available for this sort of "dynamical" analysis of complex models. One of the disadvantages of numerical integration is the large parameter and state space that have to be investigated. The (numerical) steady state 
analysis that we perform (by means of 0-isoclines) however structurizes this large search space. Moreover, we show that the number of dimensions of state spaces can be reduced (by making quasi steady state assumptions) with preservation of an interpretable correspondance of the 0-isocline plot to the model behavior. Thus, the combination of numerical integration and graphical analysis of 0-isoclines enables us to study the interesting (dynamical) properties of relatively large models without "getting lost" in confusingly large parameter and state spaces.

compartimentalization. In this model we consider only one compartment, i.e. the peritoneal cavity of a DBA/2 mouse. For macrophages this is a reasonable assumption because these cells probably do not recirculate. Macrophages enter the tissue compartment (e.g. the peritoneal cavity) from the blood compartment, and they are reported to die (although this remains uncertain) in the lymph node draining the tissue compartment [39]. T-1ymphocytes on the other hand do recirculate, and moreover they do so rapidly [40]. However, because T-lymphocytes recirculate rapidly the populations in the model can also be considered to represent the total T-lymphocyte populations (i.e. those of the whole body), since all T-1ymphocytes then travel through the peritoneal cavity often. If T-1ymphocytes do indeed recirculate rapidly, increased T-lymphocyte influx (i.e. inflammation) becomes of minor importance. INFLAM in that case however incorporates the increased production of T-lymphocyte precursor cells after an antigenic stimulation [41].

antigenicity. The relationship between tumor rejectability and tumor antigenicity, as depicted in Fig. 4, remains similar if effectors are made short-1ived. For instance, a tumor corresponding to I $2=0.2$ cannot be rejected whatever its size (if DL=0.2), whereas the tumor of Fig. 8 (I2=0.3) can be rejected when it is large. If introduced in small doses the latter tumor will however sneak through (Fig. 8a). Thus, although the form of the curve of the largest rejectable tumor (Fig. 4) remains the same, it is no longer true that tumors that are smaller than the largest rejectable tumor are also rejected.

It is important to note that the ascitic sL2 tumor grows fast (about one division every 16 hours) [20]. The fact that the mode1's immune response is "too slow" for tumor rejection may therefore depend on a fast growth rate of the tumor. The form of the relation between tumor rejectability and tumor antigenicity however remains the same for slow growing (e.g. R=0.1) tumors. The curve is shifted to the left, i.e. to tumors with a lower degree of antigenicity. However small doses of slow growing tumors, which can be rejected in large doses, 
rapidly reach a stable equilibrium (i.e. exhibit tumor dormancy [42]). This occurs independently of T-lymphocyte longevity. Sneaking through of slow growing tumors is thus absent for this particular parameter setting; however it does occur for other parameter values. CTL are more important in the reaction to slow growing tumors that to fast growing tumors because they have more time for proliferation.

implications of interactions. This model deviates from a related model investigated previously [14, 15] in two interactions: here (1) HTL restimulation depends on APC (formerly on TUMOR), and (2) CTLP differentiation depends on HTL (formerly independent). We have studied both models using an almost identical parameter setting. The study of various models for a fixed set of parameters (i.e. the multi-model fixed-parameter approach [2]), provides insight into the role of the interactions that are varied. Alternatively, a set of different models can be studied for "externally equivalent" parameter values, see Refs. [43, 44]. The behavior of the current model differs from that of the previous model in that it shows the continued T-1ymphocyte proliferation after tumor rejection. This is due to the incorporation of HTL restimulation by APC instead of by TUMOR. The incorporation of HTL factors required for the differentiation of CTLP seems to have little effect.

CTL proliferation is incorporated at the effector stage. We have also studied models that incorporate CTL proliferation at the intermediate non-cytotoxic stage. These proliferating cells mature (terminally) into the cytotoxic effector stage upon the release of HTL-derived differentiation factors [45]. The incorporation of these interactions influences the system behavior markedly: CTL populations no longer grow infinitely large because they mature into non-dividing effectors at some stage of the immune response. This can lead to "exhaustive terminal differentiation" [46] which corresponds to the theoretically described "overmaturation" [47, 48]; this results in reduced immune responses in the case of high doses of antigen. Low zone tolerance (e.g. sneaking through) however remains unaffected by the incorporation of terminal differentiation [45].

tolerance. We have shown above that in this proliferative model, in which no negative (e.g. suppressive) interactions were incorporated, unresponsiveness evolves when T-1ymphocyte precursor cells become depleted. In the case of tumors this generates the sneaking through phenomenon. In the case of antigens that do not expand infinitely (e.g. an allogenic organ graft) this corresponds to a stable equilibrium between the organ at its normal size and an immune reaction of very limited magnitude [45]. Such a tolerance state 
evolves in low zone circumstances (like sneaking through evolves here) and in "neonatal" circumstances, i.e. in immature immune systems. Depletion of helper $T$ cell precursors and the course of IL 2 production determine whether tolerance or a vigorous immune reaction develops.

\title{
ACKNOWLEDGEMENTS
}

We are grateful to Prof. Rudy Ballieux for his enthusiastic support. The participants of the workshop are thanked for their helpful criticism; which we have tried to incorporate in the present paper. We thank Miss S.M. McNab for linguistic advice.

\author{
REFERENCES
}

1. Hogeweg, P. and B. Hesper. 1981. Two predators and one prey in a patchy environment: an application of MICMAC modeliing. J. Theor. Bio1. $93: 411$.

2. De Boer, R.J. and P. Hogeweg. 1985. Tumor escape from immune elimination: simplified precursor bound cytotoxicity models. J. Theor. Biol. 113:719.

3. Herberman, R.B. 1983. Lymphoid cells in immune surveillance against malignant transformation. In: Adv. in Host Defense Mechanisms, Vol.2. Eds. J.I. Gallin and A.S. Fauci. Raven Press, New York P. 241 .

4. Steel, G.G. 1977. Growth kinetics of tumours. Clarendon Press, oxford.

5. Unanue, E.R. 1984. Antigen-presenting function of the macrophage. Ann. Rev. Immuno1. 2:395.

6. Critrom, A.A., G.H. Sunshine, T. Reme, R. Ceredig, A.L. Glasebrook, A. Kelso, and H.R. McDonald. 1983. Stimulator cell requirements for allospecific $T$ cell subsets: specialized accessory cells are required to activate helper but not cytolytic T-lymphocyte precursors. J. Immunol. 130:2.

7. Wagner, H., C. Hardt, K. Heeg, K. Pfizenmaier, W. Solbach, R. Bartlett, H. Stockinger, and M. Rblitinghoff. 1980. T-T ce11 interactions during CTL response: T cell derived helper factors (interleukin 2) as a probe to analyse CTL responsiveness and thymic maturation of CTL progenitors. Immunol. Rev. 51:215.

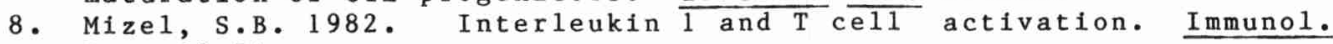
Rev. $63: 51$.

9. Van Furth, R. and D. Blusse Van Oud A1blas. 1982. The current view on the origin of pulmonary macrophages. Pathol. Res. Pract. $175: 38$.

10. Wagner, H., C. Hardt, B.T. Rouse, M. R b11inghoff, P. Scheurlich, and K. Pfizenmaier. 1982. Dissection of differentiative signals controlling murine the proliferative and responses. J. Exp. Med. 155:1876. 
11. Falk, W.; D.N. MHnne1, and W. Drbge. 1983. Activation of cytotoxic T-lymphocytes requires at least two spleen cell-derived helper factors besides interleukin 2. J. Immuno1. 130:2214.

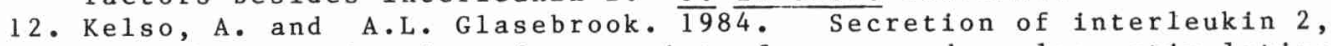
macrophage-activating factor, interferon, and colony-stimulating factor by alloreactive T-1ymphocyte clones. J. Immunol. 132:2924.

13. Van Loveren, H., K. Kato, R. Meade, D.R. Green, M. Horowitz, W. Ptak, and P.W. Askenase. 1984. Characterization of two different Ly-1 ${ }^{+} \mathrm{T}$ cell populations that mediate delayed-type hypersensitivity. J. Immuno1. 133:2402.

14. $\overline{\text { De }}$ Boer, R.J., P. Hogeweg, H.F.J. Dullens, R.A. De Weger, and W. Den Otter. 1985. Macrophage T-1ymphocyte interactions in the anti-tumor immune response: a mathematical model. J. Immunol. $134: 2748$.

15. De Boer, R.J. and P. Hogeweg. submitted. Macrophage T-1ymphocyte interactions II: sneaking through intrinsic to helper T cell dynamics.

16. Berendt, M.J. and R.J. North. 1980. T cel1-mediated suppression of anti-tumor immunity. An explanation for progressive growth of an immunogenic tumor. J. Exp. Med. 151:69.

17. Herberman, R.B. 1982. Ceis suppressing cell-mediated immune responses of cancer patients. In: Human Suppressor Cell, Ed. B. Serrou. North Holland, Amsterdam P. 179.

18. Prehn, R.T. 1982. Antigenic heterogeneity: a possible basis for progression. In: Tumor Cell Heterogeneity: Origins and Implications. Eds. A.H. Owens, D.S. Coffey, and S.B. Baylin. Academic Press, New York and London P. 73.

19. O1d, L.J., E. Stockert, E.A. Boyse, and J.H. Kim.1968. Antigenic modulation. Loss of TL antigen from cells exposed to TL antibody. Study of the phenomenon in vitro. J. Exp. Med. 127:523.

20. Dullens, H.F.J., J. Hilgers, B.J. Spit, E. De Heer, R.A. De Weger, C.D.H. Van Basten, and W. Den Otter. 1982. Staging, growth properties and metastatic behaviour of a transplantable murine T ce11 1ymphoma. Cancer Treat. Rep. 63:99.

21. Den otter, W. 1981. The effect of activated macrophages on tumor growth in vitro and in vivo. Lymphokines 3:389.

22. De Boer, R.J. 1983. GRIND: Great Integrator Differential Equations. Bioinformatics Group, University of Utrecht, The Netherlands.

23. Gottwald, B.A. and G. Wanner. 1981. A reliable Rosenbrock integrator for stiff differential equations. Computing 26:355.

24. Lannin, D.R., S. Yu, and C.F. McKahn. 1982. Thymus-dependent response: too little and too late for immune surveillance. Transplantation 33:99.

25. Du11ens, H.F.J., C. Venengoor, R.A. De Weger, F. Woutersen, R.A. Woutersen, and W. Den 0tter. 1979. Comparison of various forms of therapy in two different mouse tumour systems. Cancer Treat. Rep. $63: 99$.

26. Miller, R.A. and 0. Stutman. 1984. T cel1 repopulation from functionally restricted splenic progenitors: 10,000-fold expansion documented by using limiting dilution analysis. J. Immunol. $133: 2925$.

27. Ishii, Y., A. Matsuura, T. Takami, T. Uede, Y. Ibayashi, T. Uede, M. Imamura, K. Kikuchi, and Y. Kikuchi. 1984. Lymphoid cell subpopulations infiltrating into autologous rate tumors undergoing rejection. Cancer Res. 44:4053.

28. Ibayashi, Y. T. Uede, T. Uede, and K. Kikuchi. 1985. Functional analysis of mononuclear cells infiltrating into tumors: differential cytotoxicity of mononuclear cells from tumors of immune and nonimmune rats. J. Immunol. 134:648.

29. Jerne, N.K. 1984. Idiotypic networks and other preconceived ideas. Immuno1. Rev. $79: 5$.

30. Prehn, R.T. 1976. Immunostimulation of the lymphodependent phase 
of neoplastic growth. J. Nat1. Cancer Inst. 59:1043.

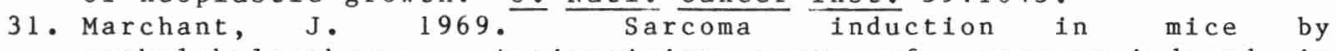
methylcholanthrene. Antigenicity tests of sarcomas induced in thymus grafted and control animals. Br. J. Cancer 23:383.

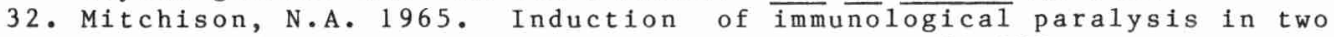
zones of dosage. Proc. R. Soc. Lond. Ser. B 161:275.

33. Weigle, W.0. 1971. Recent observations and concepts in immunological unresponsiveness and autoimmunity. Exp. Immunol. $9: 437$.

34. Malkovský, M. and P.B. Medawar. 1984. Is immunological tolerance (non-responsiveness) a consequence of interleukin 2 deficit during the recognition of antigen?. Immuno1. Today 5:340.

35. Ma1kovský, M., P. Medawar, R. Hunt, L. Palmer, and C. Doré. 1984. A diet rich in vitamin A acetate or in vivo administration of interleukin-2 can counteract a tolerogenic stimulus. Proc. R. Soc. Lond. B $220: 439$.

36. Bretscher, P. and M. Cohn. 1970. A theory of self-nonself discrimination. Paralysis and induction involve the recognition of one and two determinants on an antigen, respectively. Science $169: 1042$.

37. Teale, J.M., J.E. Layton, and G.J.V. Nossal.1979. In vitro mode1 for natural tolerance to se1f antigens. J. Exp. Med. 150:205.

38. Cleveland, R.P. and H.N. Claman. 1980. T cel1 signals: tolerance to DNFB is converted to sensitization by a separate nonspecific second signal. J. Immunol. $124: 474$.

39. Van Furth, R., M.M.C. Diesselhoff-Den Dulk, J.A. Raeburn, T.L. Van Zwet, R. Crofton, and A. Blussé Van Oud Alblas. 1980. Characteristics, origin and kinetics of human and murine mononuclear phagocytes. In: Mononuclear Phagocytes, Functional Aspects I, Ed. R. Van Furth. Martinus Nyhoff, The Hague P. 295.

40. Be11, G.I. 1978. Lymphocyte traffic patterns and ce11-cel1 interactions. In: Theoretical Immunology, Eds. G.I. Be11, A.S. Perelson, and G.H. Pimbley. Marcel Dekker, New York and Basel P. 341 .

41. Schreier, M.H. and N.N. Iscove. 1980. Hematopoietic growth factors are released in cultures of $\mathrm{H}-2$-restricted helper $\mathrm{T}$ cells, accessory cel1s and specific antigen. Nature 287:228.

42. Trainer, D.L. and E.F. Wheelock. 1984. Phenotypic shifts in the L5178Y Lymphoma during progression of the tumor-dormant state in $\mathrm{DBA} / 2$ mice. Cancer Res. 44:1063.

43. Irvine, D.H. and M.A. Savageau. 1985. Network regulation of the immune response: alternative control points for suppressor modulation of effector lymphocytes. J. Immunol. 134:2100.

44. Irvine, D.H., and M.A. Savageau. 198 $\overline{5}$. Network regulation of the immune response: modulation of suppressor lymphocytes by alternative signals including contrasuppression. J. Immuno1. $134: 2117$.

45. De Boer, R.J. and P. Hogeweg. submitted. Immunological tolerance arises due to the kinetics of IL2 production.

46. Strez1, S. 1966. Immunologica1 tolerance as the result of terminal differentiation of immunologically competent cells. Nature $209: 416$.

47. Grossman, Z. 1982. Recognition of self, balance of growth and competition: horizontal networks regulate immune responsiveness. Eur. J. Immunol. $12: 747$.

48. Grossman, Z. 1984. Recognition of self and regulation of specifity at the level of cell populations. Immunol. Rev. 79:119.

49. Merrill, S.J. 1982. Foundations of the use of an enzyme-kinetic analogy in ce11-mediated cytotoxicity. Math. Biosci. 62:219. 